EPJ manuscript No.

(will be inserted by the editor)

\title{
Motion of two micro-wedges in a turbulent bacterial bath
}

\author{
Andreas Kaiser ${ }^{1}$, Andrey Sokolov ${ }^{2}$, Igor S. Aranson ${ }^{2}$, and Hartmut Löwen ${ }^{1}$ \\ 1 Institut für Theoretische Physik II: Weiche Materie, Heinrich-Heine-Universität Düsseldorf, \\ Universitätsstraße 1, 40225 Düsseldorf, Germany \\ 2 Materials Science Division, Argonne National Laboratory, 9700 South Cass Avenue, Ar- \\ gonne, Illinois 60439, USA
}

\begin{abstract}
The motion of a pair of micro-wedges ("carriers") in a turbulent bacterial bath is explored using computer simulations with explicit modeling of the bacteria and experiments. The orientation of the two micro-wedges is fixed by an external magnetic field but the translational coordinates can move freely as induced by the bacterial bath. As a result, two carriers of same orientation move such that their mutual distance decreases, while they drift apart for an anti-parallel orientation. Eventually the two carriers stack on each other with no intervening bacteria exhibiting a stable dynamical mode where the two micro-wedges follow each other with the same velocity. These findings are in qualitative agreement with experiment on two micro-wedges in a bacterial bath. Our results provide insight into understanding selfassembly of many micro-wedges in an active bath.
\end{abstract}

\section{Introduction}

A wide variety of active suspensions $112 / 3 / 4$ are known to form remarkable spatiotemporal patterns 56617 with the appearance of coherent dynamics structures on scales that are large compared with a single self-propelled unit. Examples range from bacterial suspensions 89, spermatozoa 10]11, human crowds 12 to suspensions composed out of artificial self-propelled particles 13|14|15|16|17|18. Such systems have frequently been studied in the last year in bulk focusing on clustering [19|20|21/22 23/24, swarming [25/26/27/28 and complex swirling or turbulence [29|30|31|32]33|34|35|36. A static confinement has been shown to be able to stabilize these structures [37, accumulate and guide active particles [38/39/40/41/42/43]. This effect has been used to rectify the motion of swimmers [44|45/46/47/48] and to build sorting 49|50|51|52 as well as trapping devices 53|54|55. Furthermore the motion of passive but mobile particles submersed in an active fluid has been studied, starting with spherical and curved tracers [56/57/58] to long deformable chains [59]. Using asymmetric cogwheels a spontaneous directed rotation 6016162 can be extracted out of active bath. The translational analog is a directed motion of a single micro-wedge along its cusp induced by the active particles [6364.

In this paper, we consider micro-wedges as passive carriers and expose them to a turbulent bacterial bath. The case of a single carrier has been explored previously both by computer simulation of appropriate models resolving the individual bacteria and by 
experiments 63. For a micro-wedge kept fixed in orientation by an external uniform field and moving on a two-dimensional substrate as far as its translational motion is concerned, it was found that turbulence of the bath maximizes the directed carrier speed along its cusp. The responding mechanism was attributed to swirl depletion in the inner wedge area which gives some bacteria which are close to the wedge angle the possibility to push the carrier forward in an efficient way.

Here, we consider the case of two micro-wedges with the fixed orientation and explore by computer simulation and experiments the motion of a pair of carriers in two dimensions. This can be understood as a first step towards the hierarchical selfassembly of many carriers in a bacterial bath. We compare two different configurations of the wedges: parallel and anti-parallel orientated carriers. Since each wedge is transported in the direction of its cusp, two carriers of the same orientation move such that their mutual distance decreases, while they drift apart for an anti-parallel orientation. Eventually, the two carriers of same orientation will end up in a state where they stack closely on top of each other such that there are no intervening bacteria left. They exhibit a stable dynamical mode in which the two micro-wedges follow each other with the same speed. The distribution function of the wedge distance averaged over a finite time shows a subtle multiple-peak structure which is compatible with the swirl depletion picture. We obtain our results within the same model successfully applied to the description of a single micro-wedge by calculating the average relative velocity and the distribution of the wedge distances. Our numerical results are in qualitative agreement with experiment on two micro-wedges in a turbulent bacterial bath.

This paper is organized as follows: in Sect. 2 we will explain the used model before we present results for the motion of the micro-carriers obtained by particle resolved simulations in Sect. 3 and experiments in Sect. 4. Finally, we conclude in Sect. 5.

\section{Model}

We model the bacterial bath in two spatial dimensions by $N$ rod-like self-propelled units with an effective body shape asymmetry analogous to Ref. 63. Each rod of length $\ell$ and width $\lambda$ is discretized into $n=6$ spherical segments equidistantly positioned, with a displacement $s=0.85 \lambda$, along the main $\operatorname{rod} \operatorname{axis} \hat{\mathbf{u}}=(\cos \varphi, \sin \varphi)$, see Fig. 1. The according aspect ratio $p=\ell / \lambda=5$ is chosen in order to model Bacillus subtillis suspensions, as considered in our experiments. A repulsive Yukawa potential is imposed between the segments of different rods 65. The resulting pair potential of a rod pair $\alpha, \beta$ is given by $U_{\alpha \beta}=\sum_{i=1}^{n} \sum_{j=1}^{n} U_{i} U_{j} \exp \left[-r_{i j}^{\alpha \beta} / \lambda\right] / r_{i j}^{\alpha \beta}$, where $\lambda$ is the screening length and $r_{i j}^{\alpha \beta}=\left|\mathbf{r}_{i}^{\alpha}-\mathbf{r}_{j}^{\beta}\right|$ the distance between segment $i$ of $\operatorname{rod} \alpha$ and segment $j$ of $\operatorname{rod} \beta(\alpha \neq \beta)$. In analogy to our previous work 6366 we incorporate an effective shape asymmetry to account for the experimental observed swim-off effect of colliding bacteria 8967 . We increase the interaction prefactor for the first segment with respect to the others of each rod. This quantity is given by $U_{1}^{2} / U_{j}^{2}=3$ $(j=2 \ldots n) 63$. Any overlap of particles is prohibited by choosing a large interaction strength $U_{j}^{2}=2.5 F_{0} \ell$. Here $F_{0}$ is an effective self-propulsion force directed along the main rod axis and leading to a constant propulsion velocity $v_{0} 68$. We do not resolve details of the actual propulsion mechanism. Hydrodynamic interactions between the swimmers are neglected which is expected to be justified at high packing fractions in the absence of any global flow, i.e. in an orientationally disordered configuration as considered here [37.

Micro-swimmers move in the low Reynolds number regime. The corresponding overdamped equations of motion for the positions $\mathbf{r}_{\alpha}$ and orientations $\hat{\mathbf{u}}_{\alpha}$ are 


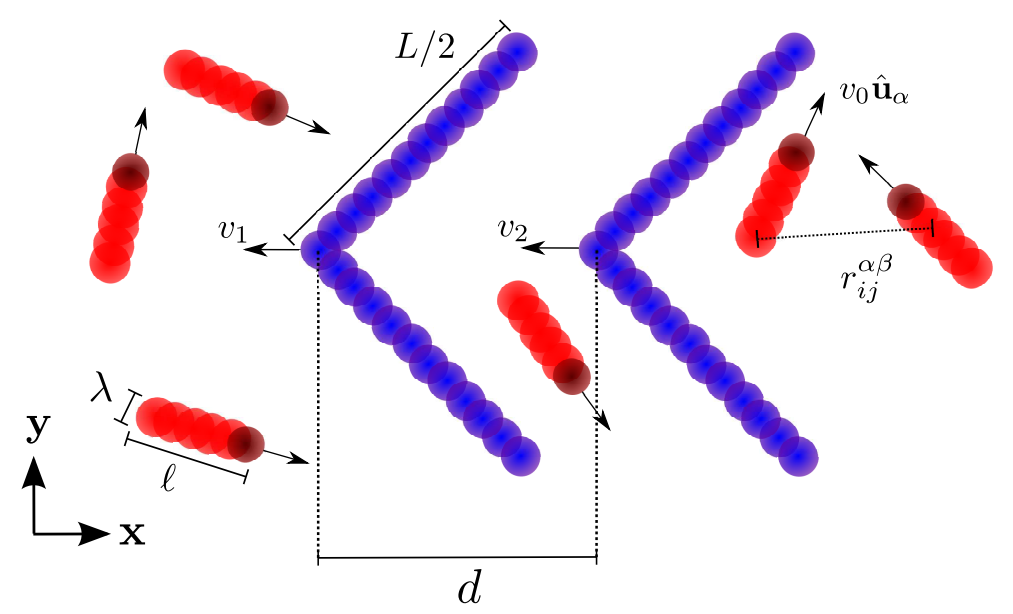

Fig. 1: Schematic representation of the system. The self-propelled rods with an aspect ratio $\ell / \lambda$ have a propulsion velocity $v_{0}$ directed along the main rod axis $\hat{\mathbf{u}}$. The single six Yukawa segments are shown by red circles, whereby the larger interaction prefactor of the first segment is indicated by darker color. The Yukawa segments of the wedgelike carriers are shown in blue. The distance between the carriers is given by $d$ and $L$ corresponds to the contour length of each carrier. On average their velocity will be directed along the cusp and are denoted by $v_{1}$ and $v_{2}$.

$$
\begin{aligned}
\mathbf{f}_{\mathcal{T}} \cdot \partial_{t} \mathbf{r}_{\alpha}(t) & =-\nabla_{\mathbf{r}_{\alpha}} U(t)+F_{0} \hat{\mathbf{u}}_{\alpha}(t), \\
\mathbf{f}_{\mathcal{R}} \cdot \partial_{t} \hat{\mathbf{u}}_{\alpha}(t) & =-\nabla_{\hat{\mathbf{u}}_{\alpha}} U(t),
\end{aligned}
$$

in terms of the total potential energy $U=(1 / 2) \sum_{\alpha, \beta(\alpha \neq \beta)} U_{\alpha \beta}+\sum_{\alpha, \gamma} U_{\alpha \gamma}$ with $U_{\alpha \gamma}$ the potential energy of $\operatorname{rod} \alpha$ with the carrier $\gamma$. The one-body translational and rotational friction tensors for the rods $\mathbf{f}_{\mathcal{T}}$ and $\mathbf{f}_{\mathcal{R}}$ can be decomposed into parallel $f_{\|}$, perpendicular $f_{\perp}$ and rotational $f_{\mathcal{R}}$ contributions which depend solely on the aspect ratio $p=\ell / \lambda[6970$ ]

$$
\begin{aligned}
\frac{2 \pi}{f_{\|}} & =\ln p-0.207+0.980 p^{-1}-0.133 p^{-2}, \\
\frac{4 \pi}{f_{\perp}} & =\ln p+0.839+0.185 p^{-1}+0.233 p^{-2} \\
\frac{\pi a^{2}}{3 f_{\mathcal{R}}} & =\ln p-0.662+0.917 p^{-1}-0.050 p^{-2} .
\end{aligned}
$$

Accordingly, the propulsion velocity is given by $v_{0}=F_{0} / f_{\|}$and sets the characteristic time unit $\tau=\ell / v_{0}$.

We model a pair of micro-wedges $\gamma, \delta$ analogous to the swimmers by tiling the wedge contour length $L=20 \ell$ into Yukawa segments and restrict their motion to translation. The wedge angle is kept rectangular. The resulting equation of motion for the carriers is

$$
\mathbf{f}_{\gamma} \cdot \partial_{t} \mathbf{r}_{\gamma}(t)=-\nabla_{\mathbf{r}_{\gamma}} U_{\gamma}(t)
$$


where $\mathbf{f}_{\gamma}$ corresponds to the hydrodynamic friction coefficient of the micro-wedge, calculated using the software package HYDRO++ [1/72] and $U_{\gamma}=(1 / 2) \sum_{\gamma, \delta(\gamma \neq \delta)} U_{\gamma \delta}+$ $\sum_{\gamma, \alpha} U_{\gamma \alpha}$ is the total interaction on a single micro-wedge.

We use a rectangular simulation domain with $L_{y}=3 L / \sqrt{2}$, an aspect ratio $L_{x} / L_{y}=3$ and an area $A=L_{x} L_{y}$ and periodic boundary conditions. The total number of rods is given by $N=A \phi / \lambda \ell$, where $\phi$ is the dimensionless packing fraction, which will be fixed to $\phi=0.5$ to achieve a turbulent bacterial bath 296373. Results are obtained for simulations with randomly chosen starting distances $d$ and statistics are being gathered over a time interval $t=1000 \tau$, using a time step $\Delta t=10^{-3} \tau$. By measuring the mean displacement along the $\mathbf{x}$-direction within a single time step, the individual velocities $v_{\gamma}$ are determined by $v_{\gamma}=\left(x_{\gamma}(t+\Delta t)-x_{\gamma}(t)\right) / \Delta t$, with $\gamma=1,2$. The obtained results and the resulting transport efficiencies for the carriers depend weakly on the packing fraction within the turbulent regime of the bacterial bath [66].

\section{Simulation results}

In the following we consider two cases. Firstly, we confine the motion of the two wedges under the constraint that they both have the same $y$-coordinate and their orientation is fixed while their $x$-coordinates can respond freely to the bacterial bath. Secondly, we only fixed their orientation but relax the constraint in the $y$-direction. In our experiment the alignment constraints were imposed by the external uniform magnetic field.

For the first case, Fig. 2(a) shows the probability distribution $P(d / \ell)$ for the distance between two micro-wedges of same orientation, using the reduced distance $d / \ell$ between the carriers, measured along the cusp of the carriers, see again Fig. 11 The distribution reveals four characteristic peaks at distances $d_{1}=1 \ell, d_{2}=6 \ell, d_{3}=23 \ell$ and $d_{4}=50 \ell$ and will be explained step by step in the following.

It is interesting to correlate the peak positions with the behavior of the transport speed difference $\Delta v=v_{2}-v_{1}$ as a function of the carrier distance $d$, see Fig. 2(b). In the absence of velocity fluctuations, a peak in $P(d / \ell)$ is expected either when the transport speed difference vanishes or when the modulus of the speed difference exhibits a local minimum. In case of a vanishing relative speed at $d=d_{0}$, the sign of the slope $\partial \Delta v /\left.\partial d\right|_{d=d_{0}}$ determines the stability of the stationary situation at $d=d_{0}$ : it is stable if $\partial \Delta v /\left.\partial d\right|_{d=d_{0}}<0$ and unstable if $\partial \Delta v /\left.\partial d\right|_{d=d_{0}}>0$. For a stable situation and in the absence of velocity fluctuations, the particle would be stuck at the distances where the velocity is vanishing resulting in a divergence of the distribution function $P(d / \ell)$ at these distances.

In Fig. 2(b) we observe indeed two zeroes at about $d_{1}=1 \ell$ and about $d=28 \ell$ which compare with the peaks at $d_{1}=1 \ell$ and $d_{3}=23 \ell$. Moreover two minima in the speed difference occur at $d_{2}=6 \ell$ and $d_{4}=50 \ell$ which clearly correlates with the second and fourth peak in $P(d / \ell)$. However, for the actual height of the peak velocity fluctuations are significant which smear out the "ideal" divergence. These are defined as $\sqrt{\left\langle\left(\Delta v / v_{0}\right)^{2}\right\rangle}$, where $\langle\ldots\rangle$ denotes a time average, and shown in Fig. 2(a) as well and reveal a non-Brownian behavior.

In detail, the first peak at $d_{1}=1 \ell$ where the two wedges stick together corresponds indeed to a stable situation. When compressing the wedges more to an even smaller distance than $d_{1}=1 \ell$, the repulsive bead forces acting between the different wedges pushes them back, while expanding the mutual wedge distance is inhibited by the osmotic pressure acting on the nearly touching wedges by the surrounding bacteria. The latter effect is similar to the strong equilibrium depletion interaction between 

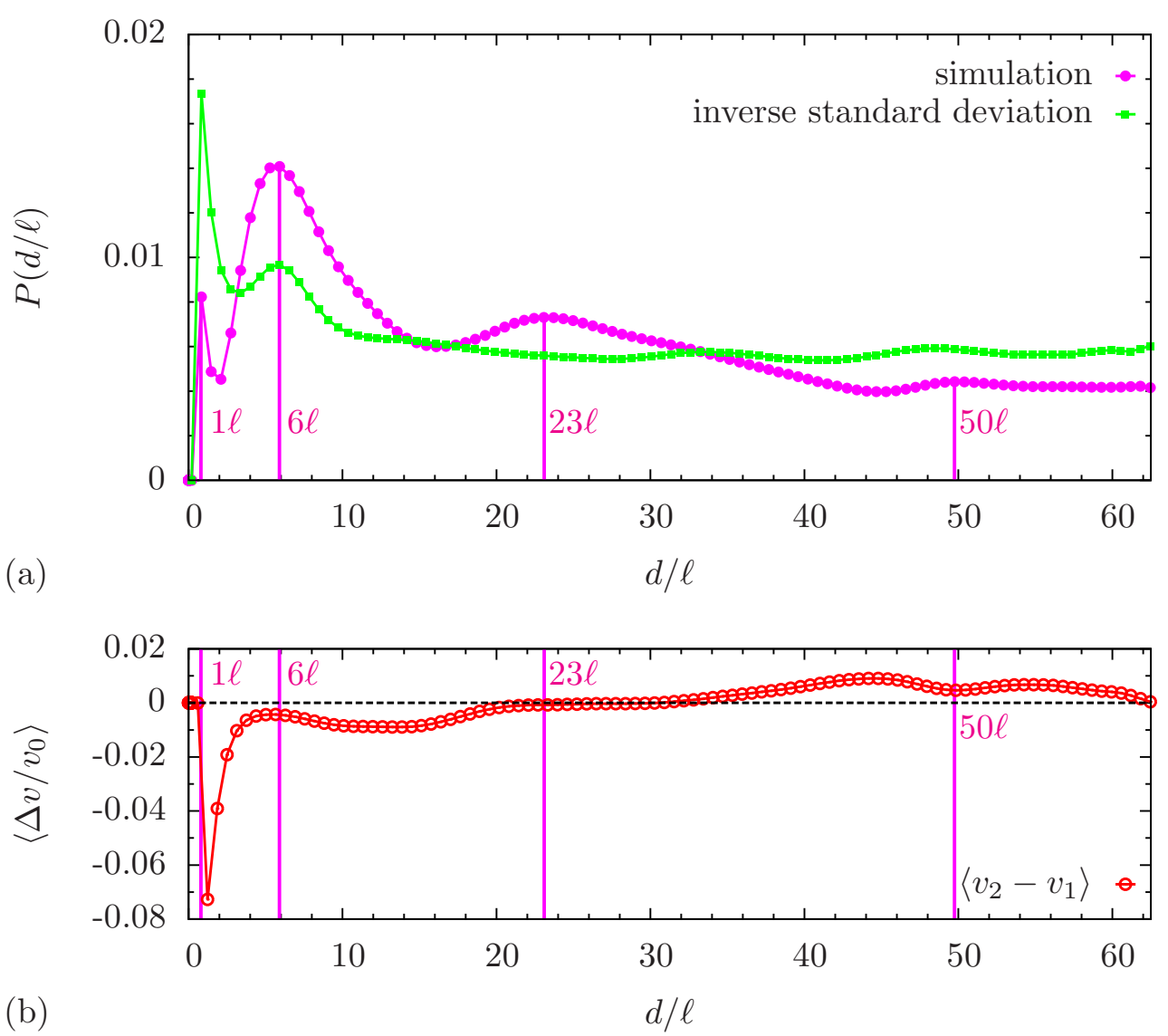

Fig. 2: (a) Probability distribution of the carrier distance $d$ measured in swimmer lengths $\ell$ obtained from simulations (magenta) and predictions due to the achieved transport efficiencies of the carriers. (b) Difference of the transport efficiencies $\Delta v=$ $v_{2}-v_{1}$ of the single carriers. Negative values mean that the right carrier is catching up, see Fig. 1. Vertical lines indicate the location of the local maxima in the probability distribution $P(d / \ell)$.

parallel rods suspended in passive spheres [74,75. In our simulations, we observed that once the wedges are sticking together at these small distances they are irreversibly bound during the time scale of the simulations such that we conclude that this is the final state of the system. Accordingly, this first peak will grow if the data are averaged over a longer simulation time when started from a randomly chosen distance.

The occurrence of the next three peaks in $P(d / \ell)$ is more subtle. In order to obtain a simple geometric picture for the second peak at $d_{2}=6 \ell$, we consider the density distribution of the bacteria around a single wedge in the frame of the moving wedge which is plotted in Fig. 3(a). This density field reveals an accumulation layer of thickness of about $a=4 \ell$ around the wedge, see Fig. 3(b), and a circular depletion zone of particles inside the wedge 63 . This depletion zone possesses a typical radius $R=7.5 \ell$ which coincides with the typical swirl size of the bulk bacterial suspension in the absence of any wedge [66. In fact, the basic idea in understanding the depletion zone is that a typical swirl swipes out particles from the inner wedge [63]. 

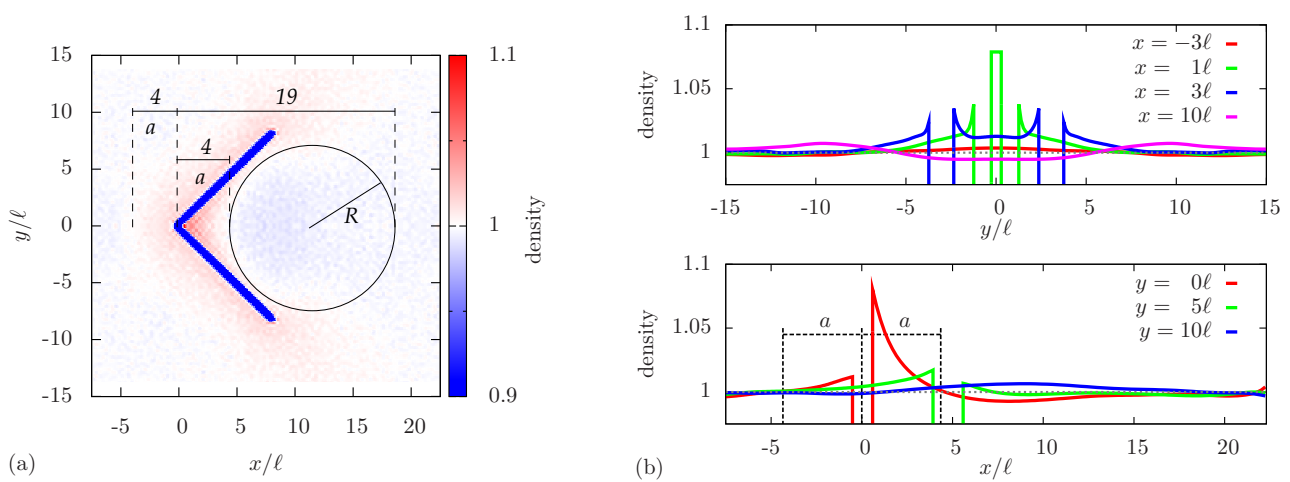

Fig. 3: (a) Density profile around a single carrier indicating the depleted wake zone. Swimmer accumulation in the cusp show possible swirl configuration. (b) Select density profiles along $\mathbf{x}$ and $\mathbf{y}$ direction through the system, showing the thickness $a$ of the bacteria accumulation layer.
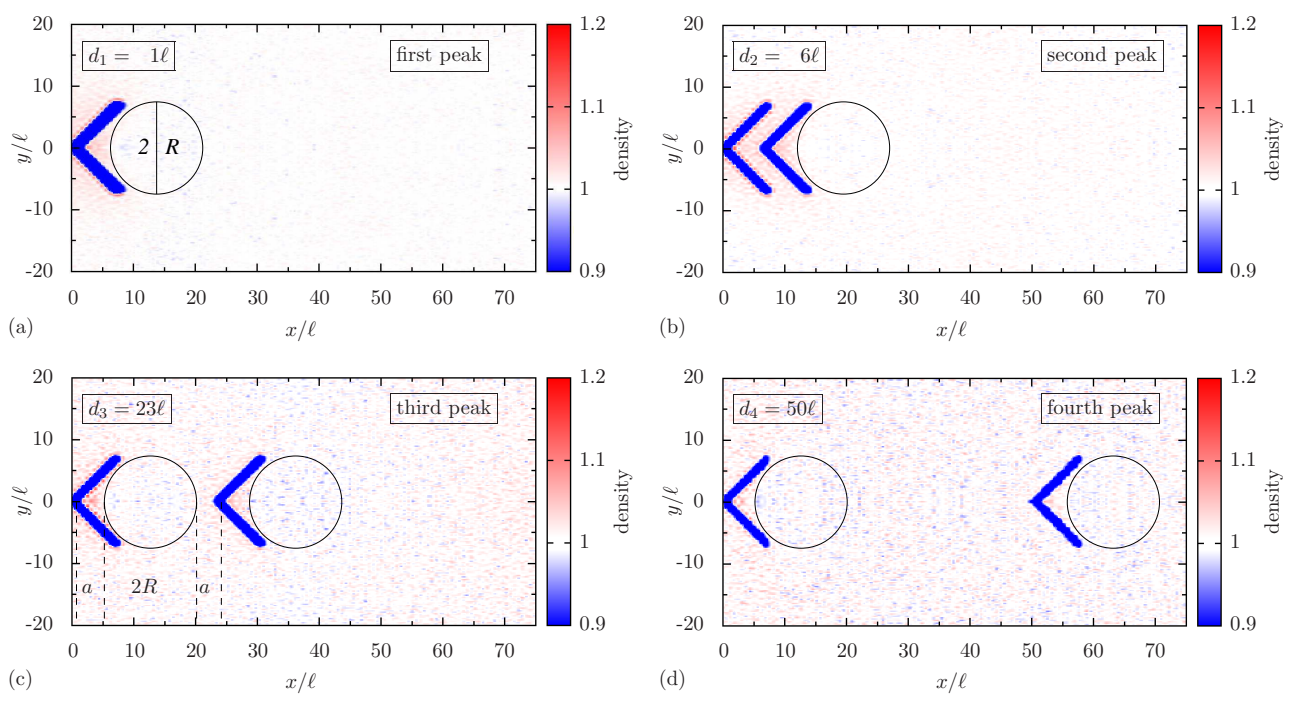

Fig. 4: Density profiles around a two carriers for four different distances $d$ between the carriers, revealing a peak in the distance probability distribution $P(d / \ell)$. Circles indicate the spatial extensions of the swirls in the wake of the carriers.

Figure 4 displays the density field around two carriers under the constraint that they are at the distances where the four peaks in $P(d / \ell)$ occur. Figure 4(a) corresponds to the first peak at $d_{1}=1 \ell$ where the two sticking wedges can hardly be distinguished and the overlapping surrounding accumulation layer responsible for the depletion attraction is clearly visible. Figure 4(b) shows the distance $d_{2}=6 \ell$ where the second peak and a speed minimum occurs. Here two stacked wedges can make use of the depletion zone causing strong interpenetration. This mutual attraction is reduced when the mutual surrounding accumulation layers of bacteria around the wedges start to overlap. This occurs roughly at a distance of $2 a=8 \ell$ which is close to the position of the second peak at $d_{2}=6 \ell$.

At a distance $d_{3}=23 \ell$ the density field is shown in Fig. 4(c). Geometrically, as also visualized in Fig. 4(c), this distance matches a swirl diameter augmented by a 


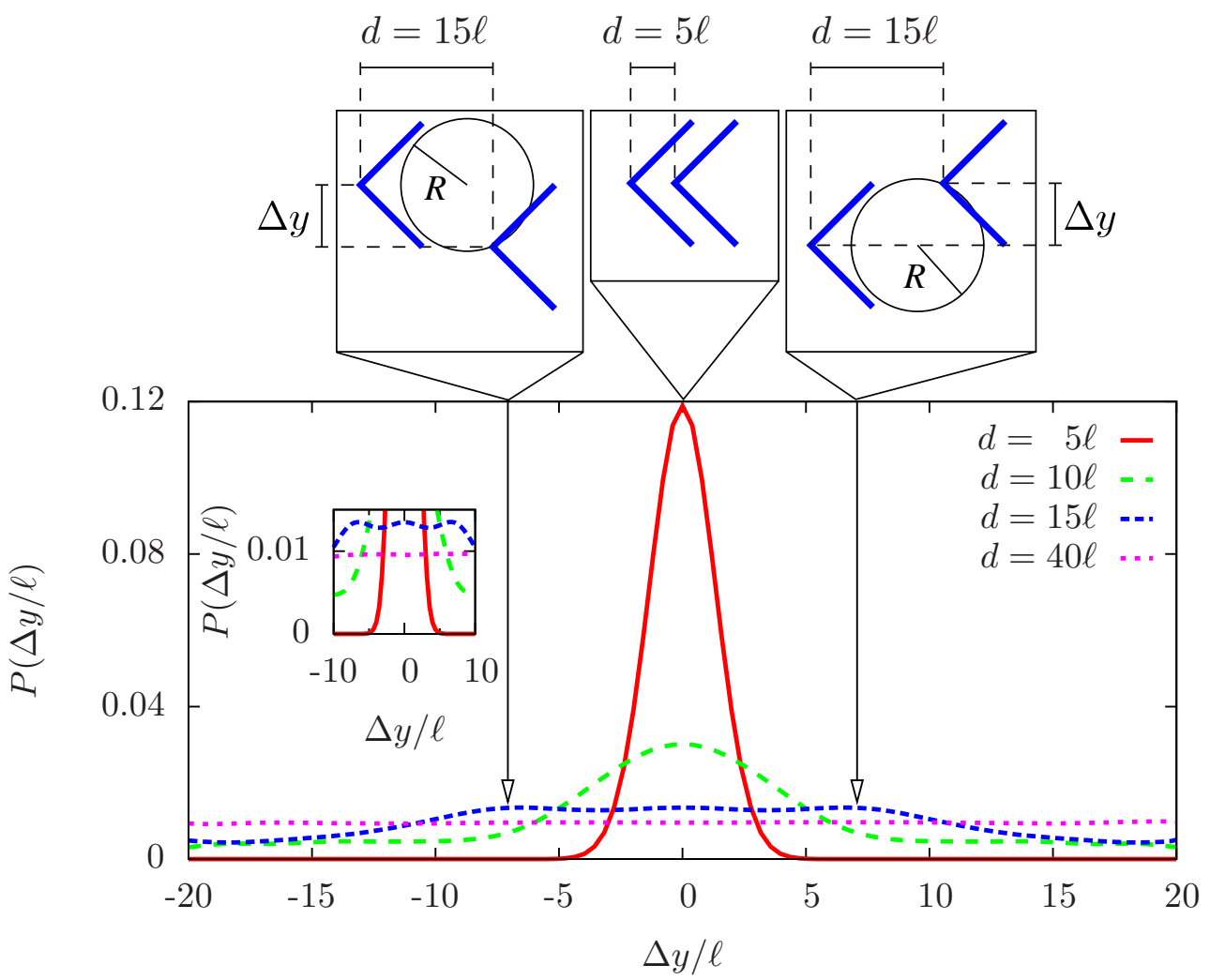

Fig. 5: Probability distribution $P(\Delta y)$ for select distances $d$. Inset shows a close up revealing the triple peak for $d=15 \ell$ and sketches indicate the configuration for the carriers for marked distances $\Delta y$.

doubled layer size $2 R+2 a=23 \ell$ and represents the unstable situation where the accumulation layer of the right wedge just starts to touch the inner swirl in the left wedge. Finally, the fourth peak at $d_{4}=50 \ell$ correlates with the occurrence of several swirls. However, in this distance regime, the variation in the relative wedge speed and the amplitude of the fourth peak are negligible.

We now analyze the second case relaxing the constraint in the $y$-direction. The probability distribution $P(\Delta y / \ell)$ to find the second carrier in the perpendicular direction $\Delta y$ is shown in Fig. 5 . Obviously, this distribution is even in $\Delta y$ due to the reflection symmetry. For large inter-carrier distances $d$ the probability is almost uniformly distributed implying that motion of two wedges is basically uncorrelated, see the data for $d=40 \ell$ in Fig. 5. As $d$ shrinks, a triple-peaked distribution $P(\Delta y / \ell)$ emerges, see the data for $d=15 \ell$ in Fig. $[5$ for which the positions of the two wedges are also explicitly indicated. The two side peaks indicate an optimal motion where the apex of the right wedge just experiences the outer range of the depletion zone, see Fig. 3(b). Finally, at closer distance the motion of the right wedge is confined within the aperture of the left one resulting in a localized distribution function $P(\Delta y / \ell)$. Again, the observed fine structure supports the general swirl depletion picture put forward in Ref. 63. 
(a)
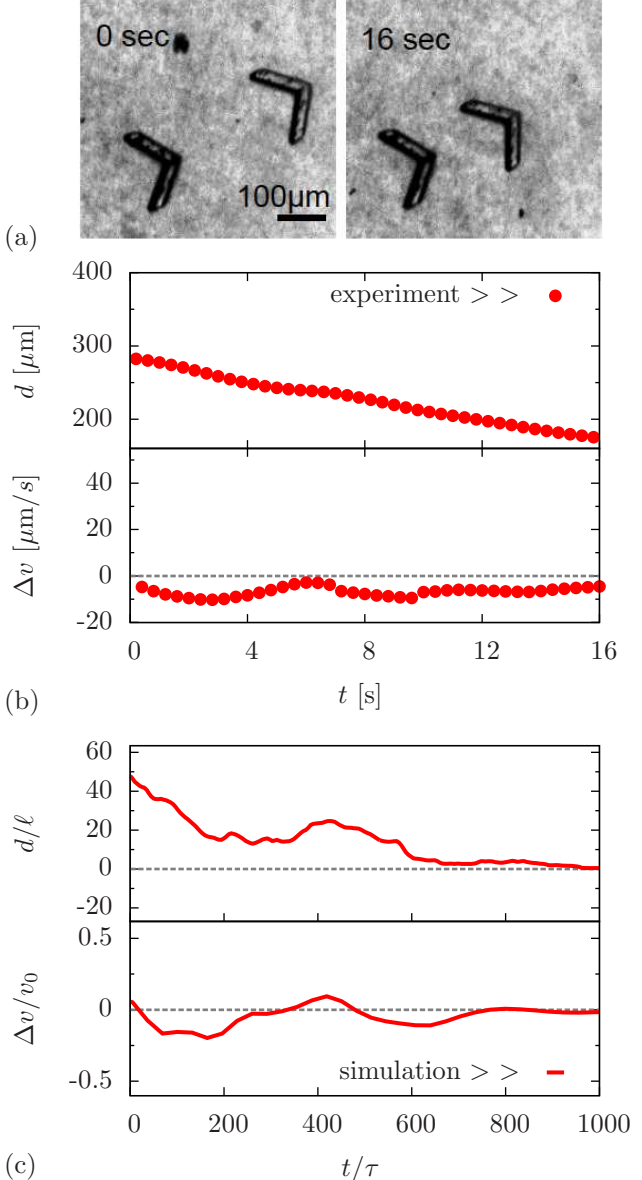
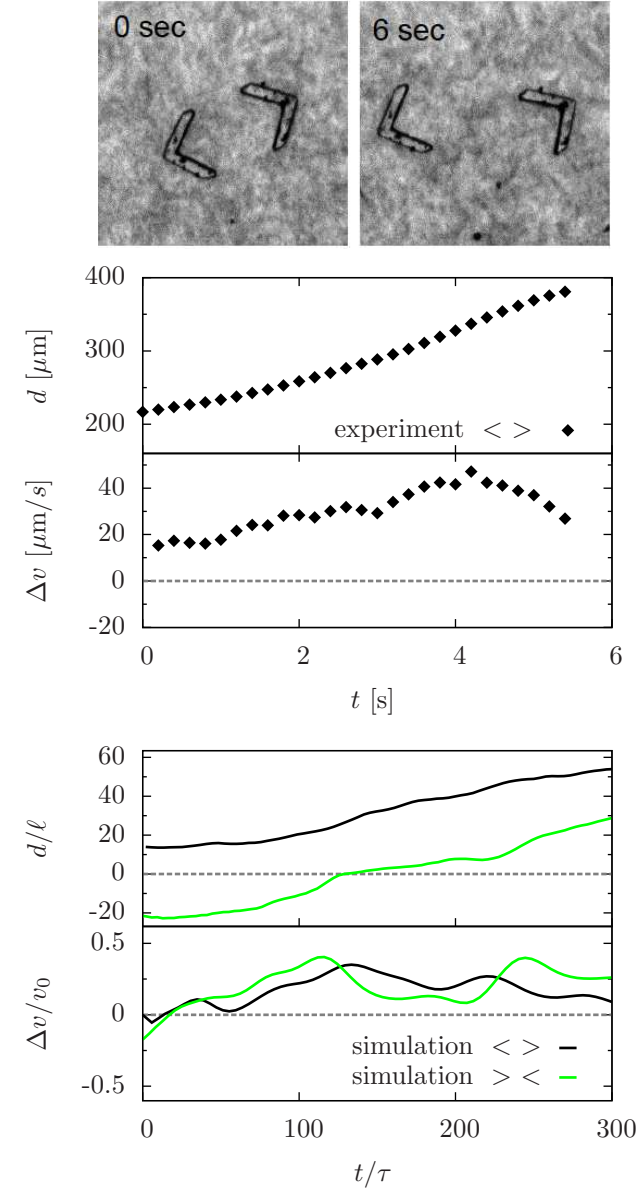

Fig. 6: (a) Illustration of the attraction and repulsion of wedge-like carriers in a turbulent bacterial bath. The orientation of the wedges is controlled by the applied uniform magnetic field. Distance $d$ between carriers and the respective relative velocity $\Delta v$ for parallel $>>$ (red) and anti-parallel orientation $\langle>$ (black) as a function of time obtained from (b) experiments and (c) simulations. A negative sign in $d$ indicates a situation of opposed wedges $><$.

\section{Experiment}

We perform series of experiments to support our theoretical findings. The microwedges were fabricated by photolithography from a mixture of photoresist and nickel particles. Nickel particles conglomerate into long chains in the course of spin coating of the mixture on a silicon wafer. After the exposure, developing, and etching, the wedgelike carriers containing small nickels particles were transferred to water by ultrasonication. Due to magnetization of the particles, the orientation of the fabricated wedges can be controlled by the external magnetic field. Two orthogonal pairs of large Helmholtz coils were used to control the orientation of the carriers. The uniform field created by these coils does not affect the positions or horizontal motions of the wedges, but only control their orientation. The micro-wedges were carefully placed into a bacterial suspension by pipetting. For this purpose the bacteria Bacillus subtilis were picked from a single colony on an agar plate and placed in a plastic tube filled 
with Terrific Broth growth medium. For optimal growth the bacteria were incubated at $35^{\circ} \mathrm{C}$ for 12 hours. Before the experiments the bacteria were extracted from the growth medium, washed and concentrated by centrifugation. The experiments were performed in a free-standing liquid film of 200-400 micron thickness, see details in Ref. 9 .

The dynamics of the wedges in the bacterial suspension was captured by Olympus IX71 microscope and digital monochrome camera (Procilica GX 1660), see Fig. 6(a). In Figs. 6(b),(c) we compare experimental and numerical results for the temporal progress of the distance between two carriers for different configurations as well as the resulting velocity difference $\Delta v=\Delta d / \Delta t$. We observed convergence of carriers of the same orientation and repulsion in the case of the opposite orientations, see Fig. 6(b), which supports our simulation results, see Fig. 6(c). At distances less than 10 micron (i.e. of the order of two bacteria length) the magnetic interaction between the shuttles dominates over hydrodynamic interaction. If the particles collide, they usually stick to each other at random orientations.

To mimic a collision of two carriers opposed in orientation $(><)$ we have performed further simulations for an initial anti-parallel configuration, see Fig. 6(c). Since each wedge is transported in the direction along its cusp, they will approach each other, collide, slide along each other and finally drift apart. Concomitantly, we observe a slow-down in the relative velocity during the collision process. We also emphasize another difference between experiment and numerical simulations. While the motion of carriers is confined in two dimensions, the motion of bacteria is threedimensional. As a result, we also observed large (compared to simulations) fluctuations in the positions of carriers due to bacterial activity. These fluctuations often prevent sticking of the carriers.

\section{Conclusions}

In line with the fascinating topic of how many passive objects self-organize in an active fluid, we have considered here the case of two micro-wedges with fixed orientations in a turbulent bacterial bath. We find an efficient stacking of the two wedges of same orientation leaving no bacteria between them. This state is a stable dynamical mode where the two micro-wedges are following each other with the same speed. There is more subtle behavior in the relative wedge motion which is compatible with the geometric swirl depletion picture put forward in Ref. 63. Our findings provide a first step towards the general case of many carriers which are therefore expected to form columnar stacks with a large persistence length reminiscent to the columnar phase of stacked bowl-shaped colloidal particles [76.

For the future study, it would be interesting to investigate the influence of hydrodynamic interactions [37] and the dynamics of submersed passive particles whose motion is non-restricted, as well as other particle shapes such as $L, C$ shapes [77/78, where stacking is also expected. However, there are also shapes where stacking is frustrated (like for $T$-shaped carriers) which are expected to form loosely-packed gels 79 . Furthermore, for future research, it would be challenging to study the motion of passive particles for gliding bacteria, where large clusters emerge [21. It is expected that such clusters have a significant influence on the dynamics of the wedges. For gliding bacteria, hydrodynamic interactions are less relevant which makes our modelling an even more appropriate one for this realization. Moreover, a microscopic theory for active depletion 80 is highly desirable to make predictions for the carrier motions which could be based on kinetic [81] or dynamical density functional theory 3982. 
A. K. was supported by the ERC Advanced Grant INTERCOCOS (Grant No. 267499) and H. L. by the science priority program SPP 1726 of the German Science Foundation (DFG). Work by A. S. and I. S. A. was supported by the U.S. Department of Energy (DOE), Office of Science, Basic Energy Sciences (BES), Materials Science and Engineering Division.

\section{References}

1. P. Romanczuk, M. Bär, W. Ebeling, B. Linder, and L. Schimansky-Geier, Eur. Phys. J. Spec. Top. 202, 1 (2012).

2. I. S. Aranson, Physics-Uspekhi 56, 79 (2013).

3. M. C. Marchetti, J. F. Joanny, S. Ramaswamy, T. B. Liverpool, J. Prost, M. RaO, and R. A. Simha, Rev. Mod. Phys. 85, 1143 (2013).

4. M. E. Cates, Rep. Prog. Phys. 75, 042601 (2012).

5. J. Toner, Y. H. Tu, and S. Ramaswamy, Ann. Phys. 318, 170 (2005).

6. T. ViCseK and A. ZAfeiris, Phys. Rep. 517, 71 (2012).

7. G. Subramanian, D. L. Koch, and S. R. Fitzgibbon, Phys. Fluids 23, 170 (2011).

8. K. Drescher, J. Dunkel, L. H. Cisneros, S. Ganguly, and R. E. Goldstein, Proc. Natl. Acad. Sci. U.S.A. 108, 10940 (2011).

9. A. Sokolov, I. S. Aranson, J. O. Kessler, and R. E. Goldstein, Phys. Rev. Lett. 98, 158102 (2007).

10. I. H. Riedel, K. Kruse, and J. Howard, Science 309, 300 (2005).

11. B. M. Friedrich and F. Jülicher, New J. Phys. 10, 123035 (2008).

12. J. L. Silverberg, M. Bierbaum, J. P. Sethna, and I. Cohen, Phys. Rev. Lett. 110, 228701 (2013).

13. D. Volfson, A. Kudrolli, and L. S. Tsimring, Phys. Rev. E 70, 051312 (2004).

14. I. S. Aranson and L. S. Tsimring, Rev. Mod. Phys. 78, 641 (2006).

15. W. Wang, W. Duan, A. Sen, and T. E. Mallouk, Proc. Natl. Acad. Sci. U.S.A. 110, 17744 (2013).

16. H.-R. Jiang, N. Yoshinaga, and M. Sano, Phys. Rev. Lett. 105, 268302 (2010).

17. L. Baraban, D. Makarov, R. Streubel, I. Mönch, D. Grimm, S. Sanchez, and O. G. SchmidT, ACS Nano 6, 3383 (2012).

18. R. Kapral, J. Chem. Phys. 138, (2013).

19. F. Peruani, A. Deutsch, and M. Bär, Phys. Rev. E 74, 030904 (2006).

20. I. Theurkauff, C. Cottin-Bizonne, J. Palacci, C. Ybert, and L. Bocquet, Phys. Rev. Lett. 108, 268303 (2012).

21. F. Peruani, J. Starruss, V. Jakovluevic, L. Søgandd-Andersen, A. Deutsch, and M. B̈̈r, Phys. Rev. Lett. 108, 098102 (2012).

22. I. Buttinoni, J. Bialké, F. Kümmel, H. Löwen, C. Bechinger, and T. Speck, Phys. Rev. Lett. 110, 238301 (2013).

23. J. Palacci, S. Sacanna, A. P. Steinberg, D. J. Pine, and P. M. Chaikin, Science 339, 936 (2013).

24. A. ZötTl and H. StARK, Phys. Rev. Lett. 112, 118101 (2014).

25. H. Zhang, A. Be'er, R. Smith, E.-L. Florin, and H. Swinney, Europhys. Lett. 7, 48011 (2009).

26. S. Thutupalli, R. Seemann, and S. Herminghaus, New J. Phys. 13, 073021 (2011).

27. T. Vicsek, A. Czirók, E. Ben-Jacob, I. Cohen, and O. Shochet, Phys. Rev. Lett. 75, 1226 (1995).

28. M. Abkenar, K. Marx, T. Auth, and G. Gompper, Phys. Rev. E 88, 062314 (2013).

29. H. H. Wensink, J. Dunkel, S. Heidenreich, K. Drescher, R. E. Goldistein, H. Löwen, and J. M. Yeomans, Proc. Natl. Acad. Sci. U.S.A. 109, 14308 (2012).

30. C. W. Wolgemuth, Biophys. J. 95, 1564 (2008).

31. S. Zhou, A. Sokolov, O. D. Lavrentovich, and I. S. Aranson, Proc. Natl. Acad. Sci. U.S.A. 111, 1265 (2014).

32. J. Dunkel, S. Heidenreich, K. Drescher, H. H. Wensink, M. Bär, and R. E. Goldstein, Phys. Rev. Lett. 110, 228102 (2013). 
33. A. Rabani, G. Ariel, and A. Be'er, PLoS ONE 8, e83760 (2013).

34. R. Grossmann, P. Romanczuk, M. BÄr, and L. Schimansky-Geier, Phys. Rev. Lett. 113, 258104 (2014).

35. J. Gachelin, A. Rousselet, A. Lindner, and E. Clement, New J. Phys. 16, 025003 (2014).

36. A. Sokolov and I. S. Aranson, Phys. Rev. Lett. 109, 248109 (2012).

37. E. Lushi, H. Wioland, and R. E. Goldstein, Proc. Natl. Acad. Sci. U.S.A. 111, 9733 (2014).

38. J. Elgeti, U. B. Kaupp, and G. Gompper, Biophys. J. 99, 1018 (2010).

39. H. H. Wensink and H. Löwen, Phys. Rev. E 78, 031409 (2008).

40. G. Li and J. X. TANG, Phys. Rev. Lett. 103, 078101 (2009).

41. P. Denissenko, V. Kantsler, D. J. Smith, and J. Kirkman-Brown, Proc. Natl. Acad. Sci. U.S.A. 109, 8007 (2012).

42. Y. Fily, A. Baskaran, and M. F. Hagan, Soft Matter 10, 5609 (2014).

43. I. D. Vladescu, E. J. Marsden, J. Schwarz-Linek, V. A. Martinez, J. Arlt, A. N. Morozov, D. Marenduzzo, M. E. Cates, and W. C. K. Poon, Phys. Rev. Lett. 113, 268101 (2014).

44. P. Galajda, J. Keymer, P. Chaikin, and R. Austin, J. Bacteriol. 189, 8704 (2007).

45. M. B. Wan, C. J. Olson Reichhardt, Z. Nussinov, and C. Reichhardt, Phys. Rev. Lett. 101, 018102 (2008).

46. F. Q. Potiguar, G. A. Farias, and W. P. Ferreira, Phys. Rev. E 90, 012307 (2014).

47. A. Ротоtsky, A. M. Hahn, and H. Stark, Phys. Rev. E 87, 042124 (2013).

48. J. Tailleur and M. E. Cates, Europhys. Lett. 86, 60002 (2009).

49. J. A. Drocco, C. J. Olson Reichhardt, and C. Reichhardt, Phys. Rev. E 85, 056102 (2012).

50. S. E. Hulme, W. R. Diluzio, S. S. Shevkoplyas, L. Turner, M. Mayer, H. C. Berg, and G. M. Whitesides, Lab Chip 8, 1888 (2008).

51. I. Berdakin, A. V. Silhanek, H. N. Moyano Cortz, V. I. Marconi, and C. A. Condat, Central Eur. J. Phys. 11, 1653 (2013).

52. A. Costanzo, J. Elgeti, T. Auth, G. Gompper, and M. Ripoll, Europhys. Lett. 107, $36003(2014)$.

53. A. Kaiser, H. H. Wensink, and H. Löwen, Phys. Rev. Lett. 108, 268307 (2012).

54. A. Guidobaldi, Y. Jeyaram, I. Berdakin, V. V. Moshchalkov, C. A. Condat, V. I. Marconi, L. Giojalas, and A. V. Silhanek, Phys. Rev. E 89, 032720 (2014).

55. L. Restrepo-Pérez, L. Soler, C. S. Martínez-Cisneros, S. Sanchez, and O. G. SCHMidT, Lab Chip 14, 1515 (2014).

56. K. C. Leptos, J. S. Guasto, J. P. Gollub, A. I. Pesci, and R. E. Goldstein, Phys. Rev. Lett. 103, 198103 (2009).

57. G. Miño, J. Dunstan, A. Rousselet, E. Clement, and R. Soto, J. Fluid Mech. 729, 423 (2013).

58. S. A. Mallory, C. Valeriani, and A. Cacciuto, Phys. Rev. E 90, 032309 (2014).

59. A. Kaiser and H. Löwen, J. Chem. Phys. 141, 044903 (2014).

60. A. Sokolov, M. M. Apodaca, B. A. Grzyboski, and I. S. Aranson, Proc. Natl. Acad. Sci. U.S.A. 107, 969 (2010).

61. R. Dileonardo, L. Angelani, D. Dellarciprete, G. Ruocco, V. Iebba, S. Schippa, M. P. Conte, F. Mecarini, F. D. Angelis, and E. D. Fabrizio, Proc. Natl. Acad. Sci. U.S.A. 107, 9541 (2010).

62. H. Li and H. P. Zhang, Europhys. Lett. 102, 50007 (2013).

63. A. Kaiser, A. Peshiov, A. Sokolov, B. ten Hagen, H. Löwen, and I. S. Aranson, Phys. Rev. Lett. 112, 158101 (2014).

64. L. Angelani and R. D. Leonardo, New J. Phys. 12, 113017 (2010).

65. T. Kirchhoff, H. Löwen, and R. Klein, Phys. Rev. E 53, 5011 (1996).

66. A. Kaiser, A. Sokolov, I. Aranson, and H. Löwen, IEEE Trans. Nanobiosci. 14, 1 (2015).

67. I. S. Aranson, A. Sokolov, J. O. Kessler, and R. E. Goldstein, Phys. Rev. E 75, 040901 (2007). 
68. F. Kümmel, B. ten Hagen, R. Wittkowski, D. Takagi, I. Buttinoni, R. Eichhorn, G. Volpe, H. Löwen, and C. Bechinger, Phys. Rev. Lett. 113, 029802 (2014).

69. M. M. Tirado, C. L. Martinez, and J. G. De la Torre, J. Chem. Phys. 81, 2047 (1984).

70. H. LöWen, Phys. Rev. E 50, 1232 (1994).

71. J. Garcia de la Torre, S. Navarro, M. C. Lopez Martinez, F. G. Diaz, and J. J. Lopez Cascales, Biophys. J. 67, 530 (1994).

72. B. Carrasco and J. Garcia de la Torre, J. Chem. Phys. 111, 4817 (1999).

73. H. H. Wensink and H. Löwen, J. Phys. Condens. Matter 24, 464130 (2012).

74. P. G. Bolhuis, A. Stroobants, D. Frenkel, and H. N. W. Lekkerkerker, J. Chem. Phys. 107, 1551 (1997).

75. R. Ni, M. A. Cohen Stuart, and P. G. Bolhuis, Phys. Rev. Lett. 114, 018302 (2015).

76. M. Marechal, R. J. Kortschot, A. F. Demirörs, A. Imhof, and M. Dijkstra, Nano Lett. 10, 1907 (2010).

77. F. Kümmel, B. ten Hagen, R. Wittkowski, I. Buttinoni, R. Eichhorn, G. Volpe, H. Löwen, and C. Bechinger, Phys. Rev. Lett. 110, 198302 (2013).

78. H. H. Wensink, V. Kantsler, R. E. Goldstein, and J. Dunkel, Phys. Rev. E 89, 010302 (2014).

79. M. Dijkstra, J. P. Hansen, and P. Madden, Phys. Rev. Lett. 75, 2236 (1995).

80. S. K. Das, S. A. Egorov, B. Trefz, P. Virnau, and K. Binder, Phys. Rev. Lett. 112, 198301 (2014).

81. Y.-L. Chou and T. Ihle, Phys. Rev. E 91, 022103 (2015).

82. R. Wittkowski and H. Löwen, Mol. Phys. 109, 2935 (2011). 